\title{
Location and coupling interval of an ectopic excitation determine the initiation of atrial fibrillation from the pulmonary veins
}

\author{
Shunsuke Kawai ${ }^{1}$, Yasushi Mukai ${ }^{1}$, Shujiro Inoue ${ }^{2}$, Daisuke Yakabe ${ }^{3}$, Kazuhiro Nagaoka ${ }^{2}$, \\ Kazuo Sakamoto ${ }^{2}$, Susumu Takase ${ }^{2}$, Akiko Chishaki ${ }^{4}$, and Hiroyuki Tsutsui ${ }^{2}$ \\ ${ }^{1}$ Japanese Red Cross Fukuoka Hospital \\ ${ }^{2}$ Kyushu University Graduate School of Medical Sciences \\ ${ }^{3}$ National Hospital Organisation Kyushu Medical Center \\ ${ }^{4}$ Fukuoka Dental College
}

July 5, 2021

\begin{abstract}
Background and Objective: Ectopic beats originating from the pulmonary vein (PV) trigger atrial fibrillation (AF). The purpose of this study was to clarify the electrophysiological determinant of AF initiation from the PVs. Methods: Pacing studies were performed with a single extra stimulus mimicking an ectopic beat in the left superior pulmonary veins (LSPVs) in 62 patients undergoing AF ablation. Inducibility of AF, effective refractory period (ERP) and conduction properties within the PVs were analyzed. Results: A single extra stimulus in LSPV induced AF in 20 patients (32\% of all patients) at the mean coupling interval (CI) of $172 \mathrm{~ms}$. A CI-dependent anisotropic conduction at the AF onset was visualized in a 3D-mapping. Onset of AF was site-specific with reproducibility in each individual. Mean ERP in LSPV in the AF inducible group was shorter than that in the AF non-inducible group (182 $\pm 55 \mathrm{~ms}$ vs $254 \pm 51 \mathrm{~ms}, \mathrm{P}<0.0001)$. LSPV ERP dispersion was greater in the AF inducible group than in the AF non-inducible group ( $45 \pm 28 \mathrm{~ms}$ vs $27 \pm 19 \mathrm{~ms}, \mathrm{P}<0.01)$. Circumferential intra-PV conduction time (IPVCT) exhibited decremental properties in response to shortening of CI, and the prolongation of IPVCT in the AF inducible site was greater than that in the AF non-inducible site $(\mathrm{P}<0.05)$ in each individual. Conclusions: Location and coupling interval of an ectopic excitation ultimately determine the initiation of AF from the PVs. ERP dispersion and circumferential conduction delay may lead to anisotropic conduction and reentry within the PVs that initiate AF.
\end{abstract}

Location and coupling interval of an ectopic excitation determine the initiation of atrial fibrillation from the pulmonary veins

Short running Title: Location and timing determine atrial fibrillation onset

Shunsuke Kawai, MD ${ }^{\text {a, b}}$; Yasushi Mukai, MD, $\mathrm{PhD}^{\mathrm{a}}$, b; Shujiro Inoue, MD, $\mathrm{PhD}^{\mathrm{b}}$; Daisuke Yakabe, $\mathrm{MD}^{\mathrm{b}}$; Kazuhiro Nagaoka, MD, $\mathrm{PhD}^{\mathrm{b}}$; Kazuo Sakamoto, $\mathrm{MD}, \mathrm{PhD}^{\mathrm{b}}$; Susumu Takase, MD, $\mathrm{PhD}^{\mathrm{b}}$; Akiko Chishaki, $\mathrm{MD}, \mathrm{PhD}^{\mathrm{c}}$; Hiroyuki Tsutsui, $\mathrm{MD}, \mathrm{PhD}^{\mathrm{b}}$

Affiliation: ${ }^{a}$ Department of Cardiovascular Medicine, Japanese Red Cross Fukuoka Hospital, Fukuoka, Japan; ${ }^{\mathrm{b}}$ Department of Cardiovascular Medicine, Kyushu University Graduate School of Medical Sciences, Fukuoka, Japan; ${ }^{\mathrm{c}}$ Department of Health Sciences, Fukuoka Dental College, Fukuoka, Japan.

\section{Funding sources}

This study was partly supported by a research grant provided from Bristol-Myers Squibb, Inc (Kyushu University Registration Code \#GAKF850098).

Conflict of Interest: Authors declare no conflict of interests for this article. 
Corresponding author: Yasushi Mukai

Address of affiliation: 3-1-1 Okusu, Minamiku, Fukuoka 815-8555, Japan.

Tel: +81-92-521-1211, Fax: +81-92-522-3066

E-mail:y_mukai@junnai.org

\section{Authorship}

S.K., Y.M., S.I., D.Y., K.N., K.S., and S.T. - conception and design or analysis and interpretation of data; S.K., Y.M., and A.C. - drafting of the manuscript or revising it critically for important intellectual content; H.T. - approval of the manuscript submitted; S.K., Y.M., S.I., D.Y., K.N., K.S., S.T., A.C., and H.T. agreement to be accountable for all aspects of the work in ensuring that questions related to the accuracy or integrity of any part of the article.

\section{ABSTRACT}

Background and Objective: Ectopic beats originating from the pulmonary vein (PV) trigger atrial fibrillation (AF). The purpose of this study was to clarify the electrophysiological determinant of AF initiation from the PVs.

Methods: Pacing studies were performed with a single extra stimulus mimicking an ectopic beat in the left superior pulmonary veins (LSPVs) in 62 patients undergoing AF ablation. Inducibility of AF, effective refractory period (ERP) and conduction properties within the PVs were analyzed.

Results: A single extra stimulus in LSPV induced AF in 20 patients (32\% of all patients) at the mean coupling interval (CI) of $172 \mathrm{~ms}$. A CI-dependent anisotropic conduction at the AF onset was visualized in a 3D-mapping. Onset of AF was site-specific with reproducibility in each individual. Mean ERP in LSPV in the AF inducible group was shorter than that in the AF non-inducible group (182 \pm 55 ms vs $254 \pm 51$ $\mathrm{ms}, \mathrm{P}<0.0001)$. LSPV ERP dispersion was greater in the AF inducible group than in the AF non-inducible group ( $45 \pm 28 \mathrm{~ms}$ vs $27 \pm 19 \mathrm{~ms}, \mathrm{P}<0.01)$. Circumferential intra-PV conduction time (IPVCT) exhibited decremental properties in response to shortening of CI, and the prolongation of IPVCT in the AF inducible site was greater than that in the $\mathrm{AF}$ non-inducible site $(\mathrm{P}<0.05)$ in each individual.

Conclusions: Location and coupling interval of an ectopic excitation ultimately determine the initiation of AF from the PVs. ERP dispersion and circumferential conduction delay may lead to anisotropic conduction and reentry within the PVs that initiate AF.

Key Words: atrial fibrillation, catheter ablation, effective refractory period, decremental properties, site specificity

\section{Introduction}

It has been demonstrated that atrial fibrillation $(\mathrm{AF})$ is triggered by ectopic beats in the pulmonary veins (PV). ${ }^{1,2}$ Since then, a number of studies regarding electrophysiological properties in the PVs have been reported. It has been demonstrated that the effective refractory period (ERP) of the PVs in patients with $\mathrm{AF}$ was shorter than that in control patients without AF, whereas the ERP of the left atrium (LA) was not significantly different. ${ }^{3}$ The ERP heterogeneity and anisotropic conduction within the PV have been also reported, which may be the crucial electrophysiological properties in the onset of $\mathrm{AF}^{4}$ In an observation of spontaneous AF firing within the PVs using a basket catheter, the coupling interval of ectopic beats initiating AF was shorter than that of ectopic beats not initiating AF. ${ }^{5}$ However, little is known about ultimate determinant factors of AF initiation and no previous studies clearly could explain the reasons why all the ectopic beats from the PVs could not trigger AF. The purpose of this study was to clarify the electrophysiological determinant factors of AF initiation from the PVs.

\section{Methods}

Study population 
This study enrolled 62 patients with drug-refractory AF referred to our institution for AF ablation; 25 patients from Kyushu University Hospital between October 2013 and December 2016, and 37 patients from Japanese Red Cross Fukuoka Hospital between September 2019 and March 2020. We enrolled only $1^{\text {st }}$ session cases and multiple session cases were excluded. The definition of paroxysmal AF and persistent AF was followed by the American College of Cardiology/American Heart Association/Heart Rhythm Society guidelines. ${ }^{6}$ In short, paroxysmal AF was defined as episodes lasting less than 7 days and self-terminated; persistent $\mathrm{AF}$ was defined as episodes lasting more than 7 days. Antiarrhythmic drugs were discontinued at five half-lives before ablation. No patients were treated with amiodarone. The presence of LA thrombi was excluded by contrast-enhanced computed tomography. When a contrast defect in the left atrial appendage was suspected, we excluded the presence of LA thrombi by transesophageal echocardiography. This study was in compliance with the principles outlined in the Declaration of Helsinki and was approved by the institutional review board for ethics at our institution, Kyushu University Hospital (approval no. 29-44). Informed consent was obtained in the form of opt-out on the web-site. (https://www.cardiol.med.kyushuu.ac.jp/research/clinical-research/).

Electrophysiological study

All patients underwent an electrophysiological study in the fasting state under conscious sedation. A 20-pole catheter was inserted through the right jugular vein (BeeAT ${ }^{\circledR}$ Japan Lifeline, Tokyo). The proximal portion of the catheter was positioned along the superior vena cava (SVC) and crista terminalis (CT), and the distal portion was positioned in the coronary sinus (CS) for pacing and internal cardioversion.

Following a trans-septal puncture under guidance with an intracardiac echocardiography catheter (5.5-10 $\mathrm{MHz}, 8 \mathrm{Fr}$, AcuNav, Biosense Webster, Diamond Bar, CA), two or three long sheaths (SL1 ${ }^{(\mathrm{r})}$, AF Division, St. Jude Medical, Minneapolis, MN) were introduced into the LA via the same trans-septal puncture site. After left atriography was performed, 20-pole circular mapping catheters (1-5-1 mm inter-electrode spacing, $20 \mathrm{~mm}$ in diameter and/or 1-3.5-1 mm inter-electrode spacing, $15 \mathrm{~mm}$ in diameter) and a 3.5 mm open-irrigated-tip ablation catheter $\left(\right.$ Navistar $^{(\mathrm{r})}$ Thermocool $^{(\mathrm{r})}$, Biosense Webster, Diamond Bar, CA or Thermocool Smarttouch ${ }^{(\mathrm{r})}$, Biosense Webster, Diamond Bar, CA or TactiCath, Abbott, St. Paul, MN) were positioned in the pulmonary veins (PVs) for PV mapping (Fig. 1 A ). In 4 patients, PV mapping was also performed with a 64-pole basket catheter Constellation $^{(\mathrm{r})}$, Boston Scientific, San Jose, CA) (Fig. 1 B ). The electrophysiological studies were performed under support of an electroanatomical mapping system with the CARTO $^{(\mathrm{r})}$ system (Biosense Webster, Diamond Bar, CA) or Ensite Velocity system (St. Jude Medical, St. Paul, MN).

\section{Stimulation protocol}

Pulmonary vein pacing was performed from a circular mapping catheter or from the proximal (bipoles 3-4 or 5-6) electrode pair of a basket catheter located in the left superior pulmonary vein (LSPV). A cardiac stimulator (SEC-5104) was used to deliver electrical impulses of 1-ms duration at an amplitude of 10V, the negative pole being connected to the distal electrode of the pacing catheter. Electrocardiographic leads and intra-cardiac electrograms filtered at 30 to $150 \mathrm{~Hz}$ were recorded simultaneously with a polygraph (Cardio LAB IT, GE Healthcare, Chicago, IL).

A single extra stimulus coupled at $400 \mathrm{~ms}$ was decremented automatically in steps of $10 \mathrm{~ms}$ to the ERP without a basic drive pacing, mimicking a spontaneous firing from the PVs and in consideration of the influence of a basic drive pacing on the ERP. ${ }^{2,7}$ Pacing stimuli were delivered at multiple sites (anterior, roof, posterior and carina). The sinus cycle length in each patient was set between 600 to $700 \mathrm{~ms}$ under administration of intravenous isoproterenol. Cases with or without AF induction following single extra stimuli were defined as AF-inducible and non-inducible group respectively. Reproducibility of AF induction with the same coupling interval was confirmed after AF termination.

The following variables were measured: 1) ERP at each pacing site in the LSPV. The ERP was defined as the longest coupling interval at which a single extra stimulus failed to capture local myocardial sleeve within the PV. 2) Circumferential intra-PV conduction time (IPVCT) was measured from the pacing artifact to 
the end of the local electrical excitation within the plane of the circular mapping catheter at each coupling interval. The time phase at which electrical potential returned to the base line was compared among the electrode pairs and the latest time phase was considered as the end of the local electrical excitation within the assessed circular plane. The electrode pair where a pacing stimulus was delivered was considered as the initiation site of the electrical excitation. 3) PV-LA conduction time was measured from the pacing artifact to the end of the local electrical excitation at the electrode pair positioned in the CS distal at each coupling interval.

In AF inducible cases, circumferential intra-PV conduction time and PV-LA conduction time in AF inducible pacing sites were compared with those in AF non-inducible pacing sites. Sequential change of intra-PV and PV-LA conduction were assessed from $+50 \mathrm{~ms}$ to the coupling interval (CI) of AF onset in the AF inducible sites, while from $+60 \mathrm{~ms}$ to $+10 \mathrm{~ms}$ of ERP in the AF non-inducible sites. Mean value was adopted in cases that multiple AF inducible sites and/or AF non-inducible sites existed.

\section{Statistical analysis}

The data are expressed as mean +- standard deviation (SD) for continuous variables and counts and percentages for categorical variables. A comparison of categorical variables between pairs of groups was carried out using the chi-square test or Fisher's exact test. A comparison of continuous variables between pairs of groups was carried out using Student $t$-test. We performed a two-way analysis of variance (ANOVA) for a comparison of the effect of multiple levels of two factors that had multiple observations at each level. All tests were two-sided, and p-values $<0.05$ were considered significant. Analyses were conducted using software program EZR.

\section{Results}

\section{Patient characteristics}

A total of 62 patients were enrolled in this study; 43 men (69\%), 19 women (31\%), and age 66+-12 years old. There were 40 paroxysmal AF patients (64.5\%) and 22 persistent AF patients (35.5\%). Six patients had structural heart diseases; hypertrophic cardiomyopathy in 2, old myocardial infarction in 2, hypertensive heart disease in 1, and sick sinus syndrome in 1. A single extra stimulus in LSPV induced AF in 50 sites in 20 patients. LSPV carina was the most prevalent AF inducible site, followed by the roof (Fig. 2A ). The mean coupling interval (CI) at the onset of AF was 172+-69 ms (Fig. 2B ).

We divided the patients into 2 groups; AF inducible group $(\mathrm{n}=20,32.2 \%)$ and AF non-inducible group $(\mathrm{n}=42,67.8 \%)$. Table 1 summarizes the baseline characteristics of these 2 groups. There was no significant difference in age, sex, or comorbidities such as hypertension and structural heart disease between the two groups. AF type (paroxysmal AF or persistent AF), $\mathrm{CHADS}_{2}$ score, left atrial diameter (LAD), left atrial volume index (LAVI), left ventricular ejection fraction (LVEF), and administered amount of isoproterenol (ISP) were also comparable between the two groups.

\section{Inducibility of $A F$ and $P V E R P$}

Mean number of pacing sites in the same case was 4.61. We compared LSPV ERP between the 2 groups. Mean LSPV ERP in the AF inducible group was significantly shorter than that in the AF non-inducible group $(182+-55 \mathrm{~ms}$ vs $254+-51 \mathrm{~ms}, \mathrm{P}<0.0001)$ (Fig. 3A ). In addition, we compared ERP dispersion of LSPVs in each case between the 2 groups. Mean LSPV ERP dispersion in the AF inducible group was significantly greater than that in the AF non-inducible group ( $45+-28 \mathrm{~ms}$ vs $27+-19 \mathrm{~ms}, \mathrm{P}<0.01)$ (Fig. 3B ). In response to the shortening of the coupling interval, intra-PV conduction gradually lost the centrifugal activation pattern and progressively elicited anisotropic conduction within the PVs, leading to AF induction (Fig. 4, Supplementary movies ). We also focused on location-specificity. The onset of AF depended on pacing sites of the PVs. Some pacing sites were AF-inducible reproducibly, but other pacing sites, even in adjacent areas, resulted in bare premature atrial contractions (Fig. 5 ). Site-specific reproducibility in each individual was $80 \%$ among 20 cases studied. Reproducibility was confirmed 1.46 times. Although most 
of the induced AF terminated spontaneously within 1-2 minutes, electrical cardioversion was required in 4 cases $(20 \%)$. Twice electrical cardioversion was performed in 2 cases, and once in the rest 2 cases.

Quantitative analysis of circumferential intra- $P V$ and $P V-L A$ conduction time

In the AF inducible cases $(\mathrm{n}=20), 14$ cases had both $\mathrm{AF}$ inducible sites and AF non-inducible sites studied in LSPVs. Circumferential IPVCT and PV-LA conduction time were evaluated both in the AF inducible and the non-inducible sites in the same individuals. In the AF inducible sites, significant prolongation of circumferential IPVCT in response to $10 \mathrm{~ms}$ shortening of CI was observed preceding the onset of AF (Fig. 6A, 7 ). In contrast, in the AF non-inducible sites, prolongation of IPVCT in response to shortening CI was consistently modest until reaching the pacing ERP (Fig. $\mathbf{6 B}, \mathbf{7}$ ). In quantification, progressive prolongation of circumferential IPVCT in the AF inducible sites was significantly greater than that in the AF non-inducible sites. In contrast, sequential prolongation of PV-LA conduction time was comparable between the AF inducible sites and the non-inducible sites (Fig. 7 ).

\section{Discussion}

\section{Main findings}

The main findings of this study were as follows; First, a single extra stimulus in the LSPV could induce AF in $32 \%$ of the studied population. Second, onset of AF depended on coupling interval of pacing stimuli and pacing site in the PV with a site-specific reproducibility in each individual. Third, LSPV ERP in the AF inducible group was shorter than that in the AF non-inducible group. Fourth, LSPV ERP dispersion in the $\mathrm{AF}$ inducible group was greater than that in the AF non-inducible group. Fifth, circumferential intra-PV conduction exhibited decremental properties in response to shortening of coupling interval, and prolongation of intra-PV conduction time in the AF inducible site was greater than that in the non-inducible site. Finally, prolongation of PV-LA conduction time was comparable between AF inducible and non-inducible sites. This is the first study demonstrating the ultimate determinant of the AF onset from the PVs.

Importance of location and coupling interval of a PV ectopy in the onset of AF

The present study demonstrated that a single ectopic stimulus elicited AF through progressive anisotropic conduction in the PVs. The induction of AF depended on the location of the ectopic stimulus. A shortcoupled ectopic stimulus reproducibly elicited $\mathrm{AF}$ at a site but not at the adjacent area. Furthermore, the induction of AF depended on the coupling interval of ectopic stimulus. From our observation, it was obvious that an initial anisotropic conduction had a determinant role for the onset of AF, which depended on the location and coupling interval of an ectopic excitation. These findings indicate an important hypothesis that an AF trigger (site) requires a surrounding initiating substrate. Numerous previous studies of AF focused on the difference of electrophysiological properties between patients with and without AF, ${ }^{3,7}$ However, no previous study investigated in detail the site-specificity of AF induction.

\section{Electrophysiological properties of the PV}

We could demonstrate the dynamic conduction pattern changes from a centrifugal activation pattern to anisotropic activation pattern in the PVs in response to the shortening of the coupling interval. PV ERP was shorter and the ERP dispersion was greater in AF inducible patients than those in AF non-inducible patients. Thus, relatively shorter ERP and its dispersion of myocardial sleeves surrounding an ectopic excitation were associated with initial anisotropic conductions and thus the AF onset from the PVs.

Previous studies of AF investigated the difference of electrophysiological properties between the distal PV and the PV-LA junction. ${ }^{4,8}$ In addition, a number of studies discussed the 'longitudinal' PV-LA conduction delay, ${ }^{3,4,7-9}$ however, no previous study quantitatively discussed the 'axial' circumferential intra-PV conduction delay.

The present study demonstrated that circumferential intra-PV conduction delay arose at the onset of AF in response to single extra stimuli, which was more evident at AF inducible site but not at AF non-inducible sites. 


\section{Possible mechanisms of anisotropic conduction within the PVs}

A previous study compared the voltage map of the PVs among the three groups; control group without AF, paroxysmal $\mathrm{AF}$ group and persistent $\mathrm{AF}$ group, and reported that low voltage area extends as $\mathrm{AF}$ stage progresses. ${ }^{7}$ Fibrotic tissue alteration may be a possible explanation for ERP dispersion and anisotropic conduction within the PVs. We previously reported that a non-PV trigger arose in or around the atrial low voltage area, which may be a similar observation considering the property of AF triggering sites. ${ }^{10}$ Another relevant observation is the relationship between electrophysiological properties in the PVs and myocardial arrangement, reported by Hocini et al. ${ }^{11}$ They reported that zones of activation delay and conduction block were related to sudden changes in fiber direction. They also reported that fiber direction at the PV ostium was more complex and disorganized than that at the distal PV. Recently, simulation tests with computational human atrial model revealed that the greatest AF inducibility occurred for cases with circular fibers at the PV ostium. ${ }^{12,13}$ Taken together, AF initiation would be determined by an initial anisotropic conduction in relations to location and coupling interval of an ectopic excitation, and surrounding histological backgrounds.

It remains an unsolved argument whether the mechanism of the AF onset is due to reentries or a focal activation with decremental conduction. ${ }^{8,14,15}$ It is difficult to confirm a reentry by entrainment study in this field due to the short duration of stable activation pattern. On the other hand, the demonstration of first few beats with identical activation pattern at the onset of AF has been considered as an implication for a reentrant mechanism. ${ }^{5}$ Our result also demonstrated a rotational/reentrant activity within a PV using a 3D mapping with a multi-polar electrode (Fig. 4 and Supplemental Movie). We also implicated reentrant mechanisms within the PVs in patients showing PV bigeminy. ${ }^{16}$ In the present study, first 3 to 4 beats following a pacing stimulus showed identical activation pattern preceding the onset of AF, suggesting an initial reentrant mechanism (Fig. 4, 5, 6 ). This activation pattern gradually collapsed afterward. Thus, we support the hypothesis that the mechanism of the AF onset may be largely due to reentrant mechanisms.

\section{Limitations}

There are several limitations to be acknowledged in the present study. First, the study population was relatively small due to the single-center, observational study. The present findings need to be confirmed in multi-center studies. Second, the evaluation with voltage mapping within the PVs was not performed. It is conceivable that low voltage area exists at or around the site where the local functional block arises. It is also conceivable that the long myocardial sleeves are related with AF induction. ${ }^{17}$ This hypothesis can be validated by the measurement of myocardial sleeve length using voltage mapping. However, some investigators reported that myocardial sleeve length was longest in patients without AF, and that myocardial sleeve length shortened as AF stage progressed. ${ }^{7}$ Third, ERP at the AF onset sites may be overestimated. In the present study, single extra stimuli with shorter coupling interval than that at the AF onset were not delivered considering that the continuation of our study might become impossible due to the persistence of $\mathrm{AF}$ as the result of the repetitive $\mathrm{AF}$ induction. ${ }^{18,19}$ If single extra stimuli were continued to deliver until coupling interval of a single extra stimulus reached the ERP, the prolongation of intra-PV conduction time in the AF inducible sites may have been greater than that of the present results, and the difference in prolongation of intra-PV conduction time between AF inducible and non-inducible sites may have been more remarkable. Fourth, site-specificity of AF induction was confirmed only in LSPV. In this study, a series of stimulation protocol added 15-20 minutes to the total procedure time. Thus, site-specificity in other PVs was not investigated in consideration of the overextension of the procedure time.

\section{Conclusions}

Location and coupling interval of an ectopic excitation determine the AF initiation from the PVs, in relations to the surrounding initiating substrates. Dispersion of ERP and dynamic changes of circumferential conduction delay within the PVs may lead to anisotropic conduction and reentry that initiate AF.

\section{Data availability}

All data generated or analyzed during this study are included in this published article and its Supplementary 
Information files.

\section{References}

1. Häissaguerre M, Jaïs P, Shah DC, et al. Spontaneous initiation of atrial fibrillation by ectopic beats originating in the pulmonary veins. N Engl J Med 1998; 339: 659-66.

2. Chen SA, Hsieh MH, Tai CT, et al. Initiation of atrial fibrillation by ectopic beats originating from the pulmonary veins: electrophysiological characteristics, pharmacological responses, and effects of radiofrequency ablation. Circulation 1999; 100: 1879-86.

3. Jaïs P, Hocini M, Macle L, et al. Distinctive electrophysiological properties of pulmonary veins in patients with atrial fibrillation. Circulation 2002; 106: 2479-85.

4. Kumagai K, Ogawa M, Noguchi H, Yasuda T, Nakashima H, Saku K. Electrophysiologic properties of pulmonary veins assessed using a multielectrode basket catheter. J Am Coll Cardiol 2004; 43: 2281-9.

5. Arentz T, Haegeli L, Sanders P, et al. High-density mapping of spontaneous pulmonary vein activity initiating atrial fibrillation in humans. J Cardiovasc Electrophysiol 2007; 18: 31-8.

6. January CT, Wann LS, Alpert JS, et al. 2014 AHA/ACC/HRS guideline for the management of patients with atrial fibrillation: executive summary: a report of the American College of Cardiology/American Heart Association Task Force on Practice Guidelines and the Heart Rhythm Society: developed in collaboration with the Society of Thoracic Surgeons. J Am Coll Cardiol 2014; 64 (21): 2246-80.

7. Teh AW, Kistler PM, Lee G, et al. Electroanatomic properties of the pulmonary veins: slowed conduction, low voltage and altered refractoriness in AF patients. J Cardiovasc Electrophysiol 2011; 22: 1083-91.

8. Arora R, Verheule S, Scott L, et al. Arrhythmogenic substrate of the pulmonary veins assessed by high-resolution optical mapping. Ciculation 2003; 107 (13): 1816-21.

9. Tada H, Oral H, Ozaydin M, et al. Response of pulmonary vein potentials to premature stimulation. J Cardiovasc Electrophysiol 2002; 13: 33-7.

10. Kawai S, Mukai Y, Inoue S, et al. Non-pulmonary vein triggers of atrial fibrillation are likely to arise from low-voltage areas in the left atrium. Sci Rep 2019; 9: 12271.

11. Hocini M, Ho SY, Kawara T, et al. Electrical conduction in canine pulmonary veins: electrophysiological and anatomic correlation. Circulation 2002; 105: 2442-8.

12. Roney CH, Bayer JD, Dubois R, et al. The combination of pulmonary vein electrophysiology and atrial fibrosis determines driver location. Computing in Cardiology 2017; 44: 1-4.

13. Roney CH, Bayer JD, Cochet H, et al. Variability in pulmonary vein electrophysiology and fibrosis determines arrhythmia susceptibility and dynamics. PLoS Comput Biol 2018; 14 (5): e1006166.

14. Po SS, Li Y, Tang D, et al. Rapid and stable re-entry within the pulmonary vein as a mechanism initiating paroxysmal atrial fibrillation. J Am Coll Cardiol 2005; 45: 1871-7.

15. Zhou S, Chang CM, Wu TJ, et al. Nonreentrant focal activations in pulmonary veins in canine model of sustained atrial fibrillation. Am J Physiol Heart Circ Physiol 2002; 283: H1244-H1252.

16. Mukai Y, Kawai S, Inoue S, et al. Bigeminal potentials in the pulmonary vein indicate arrhythmogenic trigger of atrial fibrillation. J Arrhythm 2021; 37: 331-7.

17. Tagawa M, Higuchi K, Chinushi M, Washizuka T, Ushiki T, Ishihara N. Myocardium extending from the left atrium onto the pulmonary veins: a comparison between subjects with and without atrial fibrillation. Pacing Clin Electrophysiol 2001; 24 (10): 1459-63.

18. Allessie M, Ausma J, Schotten U. Electrical, contractile and structural remodeling during atrial fibrillation. Cardiovascular Research 2002; 54: 230-46.

19. Rostock T, Steven D, Lutomsky B, et al. Atrial fibrillation begets atrial fibrillation in the pulmonary veins. J Am Coll Cardiol 2008; 51: 2153-60.

Table 1. Patient characteristics 


\begin{tabular}{|c|c|c|c|c|}
\hline Characteristics & Total $(n=62)$ & $\begin{array}{l}\text { AF inducible ( } \\
=20)\end{array}$ & $\begin{array}{l}\text { AF non- } \\
\text { inducible }(n= \\
42)\end{array}$ & $\mathbf{p}$ \\
\hline Age (y) & $62.3 \pm 11.1$ & $67.6 \pm 11.5$ & $65.0 \pm 12.3$ & 0.44 \\
\hline Male & $43(69.3)$ & $14(70.0)$ & $29(69.0)$ & 1.0 \\
\hline Hypertension & $40(64.5)$ & $13(65.0)$ & $27(64.3)$ & 1.0 \\
\hline $\begin{array}{l}\text { Structural } \\
\text { heart disease }\end{array}$ & $6(9.7)$ & $1(5.0)$ & $5(11.9)$ & 0.65 \\
\hline CHADS2 score & $1.4 \pm 1.2$ & $1.6 \pm 1.4$ & $1.2 \pm 1.1$ & 0.27 \\
\hline LAD (mm) & $39.6 \pm 6.0$ & $41.4 \pm 7.2$ & $38.7 \pm 5.2$ & 0.1 \\
\hline LAVI $\left(\mathrm{ml} / \mathrm{m}^{2}\right)$ & $38.9 \pm 13.0$ & $40.4 \pm 13.8$ & $38.2 \pm 12.7$ & 0.56 \\
\hline LVEF (\%) & $64.6 \pm 7.3$ & $66.1 \pm 7.8$ & $63.9 \pm 7.1$ & 0.27 \\
\hline Paroxysmal AF & $40(64.5)$ & $12(60.0)$ & $28(66.7)$ & 0.78 \\
\hline $\mathbf{I} \Sigma \Pi(\mu \gamma)$ & $1.6 \pm 1.1$ & $1.5 \pm 1.1$ & $1.7 \pm 1.1$ & 0.64 \\
\hline
\end{tabular}

ISP: isoproterenol, LAD: left atrial diameter, LAVI: left atrial volume index, LVEF: left ventricular ejection fraction

\section{Figure legends}

Fig. 1. Catheter position during pulmonary vein pacing.A: 20 poles circular mapping catheters are positioned in LSPV and LIPV. B: 64 poles basket catheter is positioned in LSPV and 20 poles circular mapping catheter is positioned in LIPV.

CS: coronary sinus, HRA: high right atrium, LIPV: left inferior pulmonary vein, LSPV: left superior pulmonary vein, RV: right ventricle, SVC: superior vena cava.

Fig. 2. Details of pacing-induced AF. A: Distribution of AF inducible sites. 3D mapping shows LSPV. Each yellow dot indicates an AF inducible case. B: Histogram of coupling interval of pacing stimuli that induced $\mathrm{AF}$.

Fig.3. Difference of mean LSPV ERP and LSPV ERP dispersion between AF inducible and non-inducible groups. A: Histogram of LSPV ERP. The black bar indicates the AF non-inducible group and the gray bar indicates the AF inducible group. B: Box-and-whisker plot of LSPV ERP dispersion.

Fig. 4. Changes of conduction pattern within the PVs in response to the shortening of coupling interval. A: Changes of intra-cardiac electrogram. In this case, AF was induced when a single extra stimulus in the LSPV carina was delivered with a coupling interval of $200 \mathrm{~ms}$. Compared with the intra-cardiac electrogram with longer coupling intervals, remarkable anisotropic conduction was observed at the onset of AF. B: Ensite activation mapping of a pacing beat using a 64 electrodes basket catheter in the LSPV and a 20 electrodes circular mapping catheter in the LIPV. Shortening of the coupling interval progressively elicited anisotropic conduction within the PVs, which gave rise to the onset of AF with the coupling interval of $200 \mathrm{~ms}$.

Fig. 5. Location specificity of $\mathrm{AF}$ induction. In this case, AF was induced reproducibly with the coupling interval (CI) of $200 \mathrm{~ms}$ when a single extra stimulus was delivered in the LSPV anterior (pole 5/6). Only bare PAC could be generated even with the same CI in the adjacent area (pole $3 / 4$ or pole $7 / 8$ ).

PAC: premature atrial contraction

Fig. 6. Changes of circumferential intra-PV conduction time and PV-LA conduction time in response to shortening of coupling interval (CI). Red dots indicate the end of the local conduction. A:When a single extra stimulus was delivered in the AF inducible site (LSPV roof), significant prolongation of intra-PV conduction time was observed at the onset of AF (from $119 \mathrm{~ms}$ to $172 \mathrm{~ms}$ ) whereas PV-LA conduction time was prolonged mildly (from $187 \mathrm{~ms}$ to $217 \mathrm{~ms}$ ).B: When a single extra stimulus was delivered in the AF 
non-inducible site (LSPV antero-carina) in the same case as Fig. 5A, both intra-PV conduction time and PV-LA conduction time consistently showed mild prolongation until the CI reached ERP (270 ms in this case).

Fig. 7. Sequential changes of conduction time in response to $10 \mathrm{~ms}$ shortening of coupling interval (CI). Assessed range is from CI of $+50 \mathrm{~ms}$ to the $\mathrm{CI}$ of $\mathrm{AF}$ onset in the AF inducible sites, whereas from CI of $+60 \mathrm{~ms}$ to shortest $\mathrm{CI}$ of $\mathrm{PV}$ capture $(+10 \mathrm{~ms}$ of ERP) in the AF non-inducible sites

\section{Legend for supplemental movie}

Ensite propagation mappings of pacing beats using a 64 electrodes basket catheter in LSPV in a patient with AF. A single extra stimulus in the LSPV carina (pole G5/6) was delivered with a coupling interval from $280 \mathrm{~ms}$ to $230 \mathrm{~ms}$ in steps of $10 \mathrm{~ms}$. Shortening of the coupling interval progressively elicited anisotropic conduction within the PVs, which gave rise to the onset of AF with the coupling interval of $230 \mathrm{~ms}$.

\section{Hosted file}

Figures for JCE.pdf available at https://authorea.com/users/423749/articles/529009-locationand-coupling-interval-of-an-ectopic-excitation-determine-the-initiation-of-atrialfibrillation-from-the-pulmonary-veins 